\title{
ECOSAR: P-BAND DIGITAL BEAMFORMING POLARIMETRIC AND SINGLE PASS INTERFEROMETRIC SAR
}

\author{
Instrument Description and First Results
}

\author{
Rafael F. Rincon, Temilola Fatoyinbo, Batuhan Osmanoglu, Seungkuk Lee, K. Jon Ranson, Guoqing Sun, \\ Martin Perrine, and Cornelis Du Toit \\ NASA/Goddard Space Flight Center, \\ Greenbelt, MD 20771
}

\begin{abstract}
EcoSAR is a state-of-the-art beamforming synthetic aperture radar (SAR) recently developed at the NASA/ Goddard Space Flight Center (GSFC) for the measurement of ecosystem structure and biomass. The airborne instrument operates at a center frequency of $435 \mathrm{MHz}$ (P-band), and uses a multi-channel reconfigurable architecture to implement fully polarimetric and "single pass" interferometric measurements. The instrument architecture allows for the real-time configuration radar parameters, including center frequency, resolution, incidence angle, and number of beams, among others. The system is also designed to operate in standard or ping pong interferometric modes, and in full, orthogonal, or hybrid polarimteric modes. The instrument development was recently completed, and its first flight campaign successfully conducted in March 2014 over areas of Bahamas and Costa Rica .
\end{abstract}

Keywords - Digital Beamforming, SAR; InSAR; PolInSAR; Polarimetry, P-band.

\section{INTRODUCTION}

EcoSAR is an new beamforming synthetic aperture radar (SAR) instrument developed at the NASA/ Goddard Space Flight Center (GSFC) part of NASA's Earth Science Technology Office (ESTO) Instrument Incubator Program (IIP). The instrument is designed to make polarimetric and "single pass" interferometric measurements of ecosystem structure and biomass [1][2] on board a P3 aircraft (see Figure 1). The instrument employs an advanced wideband multichannel radar architecture that allows considerable measurement flexibility and enables imaging techniques beyond the capabilities of conventional SAR systems [3].

EcoSAR operates in the P-band with a center frequency of $435 \mathrm{MHz}$ (69 cm wavelength) at four polarizations (HH, VV, $\mathrm{HV}$, and $\mathrm{VH}$ ) using two array antennas. The EcoSAR signals propagate through vegetation structure providing information about the vegetation's three-dimensional distribution and density. Thus, EcoSAR has the unique capability of mapping above ground structure and biomass, disturbance from deforestation and degradation, forest recovery, and wetland inundation. EcoSAR measurements are also suitable for several other critically important science applications, including permafrost change, soil moisture, ice dynamics, glacier depth, and crop and land cover monitoring. The EcoSAR measurements directly support science studies recommended by the National Science Foundation's Decadal Survey [4] and highlighted in NASA's Plan for a Climate-Centric Architecture [5].

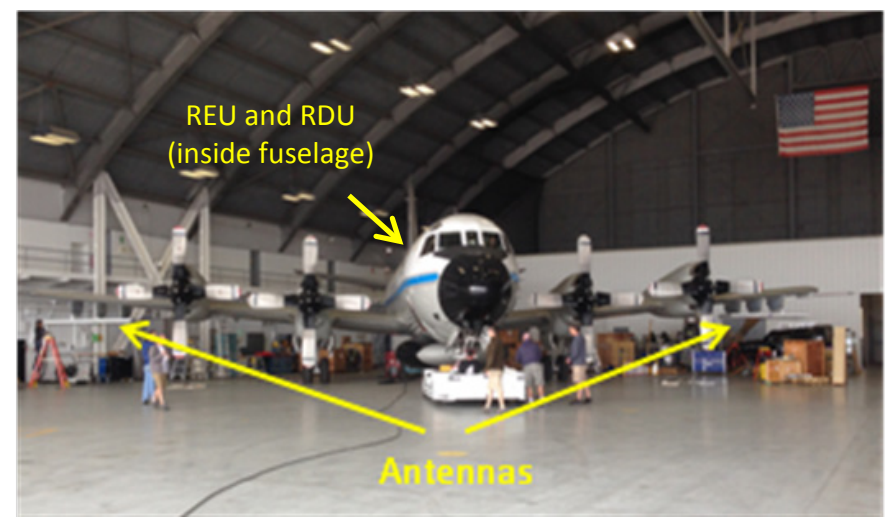

Fig. 1 The EcoSAR instrument, designed to fly on a P3 aircraft, is a polarimetric and single pass interferometric SAR instrument that employs an advanced multichannel digital and analogue architecture and two wingmounted array antennas with a baseline of $25 \mathrm{~m}$. (REU: Radar Electronics Unit, RDU: Radar Digital Unit)

EcoSAR's first flight campaign took place in March 2014 over areas of the Bahamas and Costa Rica. The instrument flew 19 hours (including transit) onboard the National Oceanic and Atmospheric Agency ( NOAA) WP-3D Orion "Hurricane Hunter" aircraft, shown in Fig. 1. The aircraft was specifically commissioned for EcoSAR's flight certification, tests, and science campaign. During the campaign, EcoSAR operated in several experimental modes and acquired data for calibration, performance assessment, and science analysis.

\section{INSTRUMENT ARCHITECTURE}

EcoSAR's architecture builds upon the L-band Digital Beamforming SAR (DBSAR) and the second generation DBSAR (DBSAR-2) designs [3][5] to perform beam steering on transmit and digital beamforming on receive. The instrument employs a 32-channel reconfigurable architecture to implement full polarimetry and single pass interferometry. Each set of 8 radar channels operates a fixed polarization (horizontal or vertical) on either side aircraft, as illustrated in Fig. 2.

The instrument's main subsystems consist of the Radar Digital Unit, the Radar Electronics Unit, and the antennas. The Radar Digital Unit (RDU) is a custom FPGA-based processor capable of multi-channel arbitrary waveform generation, data acquisition, and onboard processing. The Radar Electronics Unit (REU) is made up 32 transmit/receive (T/R) modules that conditioned the transmit and receive signals and provide 
several calibrations schemes. The antenna subsystem consists of two dual-polarization array antennas with an interferometric baseline of $25 \mathrm{~m}$. A more detailed description of each subsystem is given in the section III.

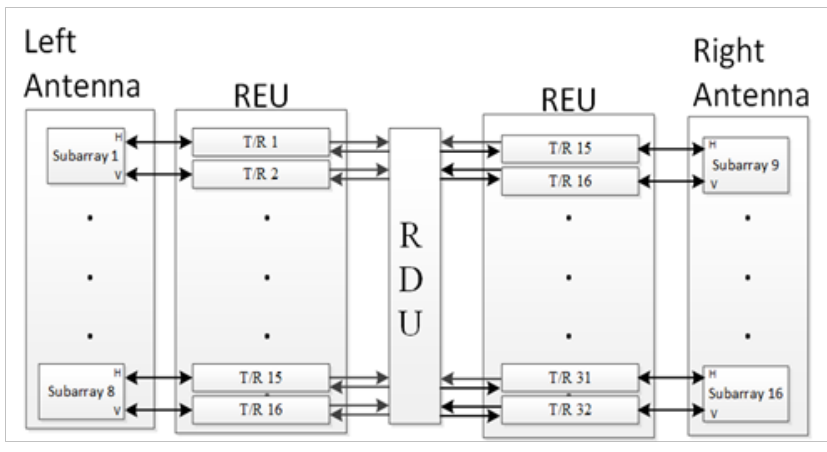

Figure 2 EcoSAR's multi-channel beamforming architecture

EcoSAR's architecture allows considerable measurement flexibility including real-time selection of the radar center frequency, bandwidth (across-track resolution), pulse length, and pulse repetition frequency (PRF). It also allows for the generation of customized transmit and receive beams, imaging both sides of the flight track (simultaneously or time interleaved), post processing synthesis of multiple beams, variable incidence angle, swath width and ground resolution. In addition, the system can operate in standard or ping pong interferometric modes, and in full, orthogonal, or hybrid polarimteric modes. A passive sensing ("sniffing") mode is also implemented to detect Radio Frequency Interference (RFI). To generate a transmit beam with particular beam characteristics and look angle, the radar generates independent waveforms with predefined amplitude and phase for each of the radar channels used. On receive, beams are digitally beamformed to achieve the desired beam characteristics and look angle.

Performance parameters and characteristics achieved with this architecture are listed on Table 1.

Table 1 EcoSAR's main characteristics

\begin{tabular}{|l|c|}
\hline Center Frequency & $435 \mathrm{MHz}^{*}$ \\
\hline Maximum Bandwidth & Up to $200 \mathrm{MHz}$ \\
\hline Polarization & $\mathrm{HH}, \mathrm{HV}, \mathrm{VH}, \mathrm{VV}$ \\
\hline Polarization Isolation & $>30 \mathrm{~dB}$ \\
\hline Noise Equivalent $\sigma_{\mathrm{o}}$ & $-41 \mathrm{~dB} *$ \\
\hline Total Number Channels & $32(8$ per polarization $)$ \\
\hline Interferometric baseline & $25 \mathrm{~m}$ \\
\hline Pulse Length & $1 \mathrm{usec}-50 \mathrm{usec}$ \\
\hline Array Peak Power/polarization & $40 \mathrm{Watts}$ \\
\hline PRF & $100 \mathrm{~Hz}-10 \mathrm{KHz}$ \\
\hline Swath & $4 \mathrm{~km} *$ \\
\hline Finest Range Resolution & $0.75 \mathrm{~m}$ \\
\hline Single Look Azimuth Resolution & $0.5 \mathrm{~m}$ \\
\hline Vertical Accuracy & $<5 \mathrm{~m}$ \\
\hline \multicolumn{2}{|c}{$*$ nominal operation }
\end{tabular}

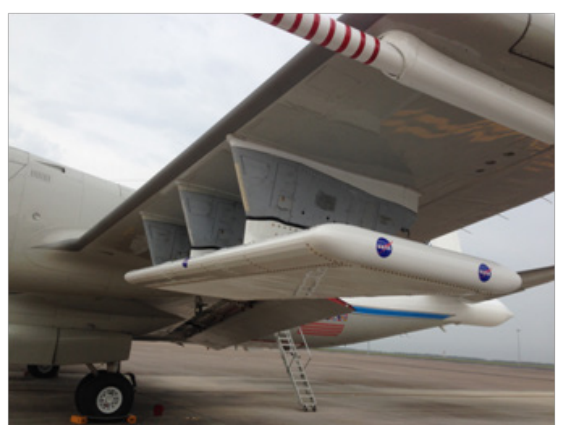

Fig. 3 EcoSAR antennas (only one shown) install under the P3 aircraft wings permiting field of view on both sides of the aircraft and providing a $25 \mathrm{~m}$ interferometric baseline.

\section{SYSTEM DESCRIPTION}

\section{A. Antennas}

The EcoSAR antennas are based on a custom stacked-patch design that permits polarimetric radar operation with bandwidths up to $120 \mathrm{MHz}$ in the horizontal polarization and $200 \mathrm{MHz}$ in the vertical polarization. Each antenna is made up of 10 dual-polarized sub-arrays. Only the eight central arrays are energized. The sub-arrays are enclosed in an aerodynamic fairing designed and built by the Department of Aerospace Engineering at the University of Kansas [6]. The antennas are installed on each side of the aircraft (see Fig. 1) under the aircraft wings using pylons and customized extenders, as shown in Fig. 3. This configuration results in a separation between the centers of the two antennas (baseline) of $25 \mathrm{~m}$.

\section{B. Radar Electronic Unit (REU)}

The REU consists of dedicated T/R modules for each of the 32 radar channels, power distribution units and a thermal control unit. Each T/R module conditions the transmit and receive signals, and performs several real-time calibration schemes, including independent transmit and receive channel calibration, loopback calibration, and antenna VSWR measurements as a function of look angle. Timing and logic signals for the modules operation is provided by the RDU.

The $\mathrm{T} / \mathrm{R}$ modules and power supplies are installed on aircraft racks, which reside inside the aircraft fuselage, as shown in Fig. 4. Each T/R module includes a 20-Watt solid-state power amplifier (SSPA), low noise amplifier (LNA), circulator, coupler, filters, and control switches. The transmit path was designed accounting for dynamic range, impedance matching and signal levels. The receive path was designed in a similar fashion but noise figure and out-ofband rejection had to be considered.

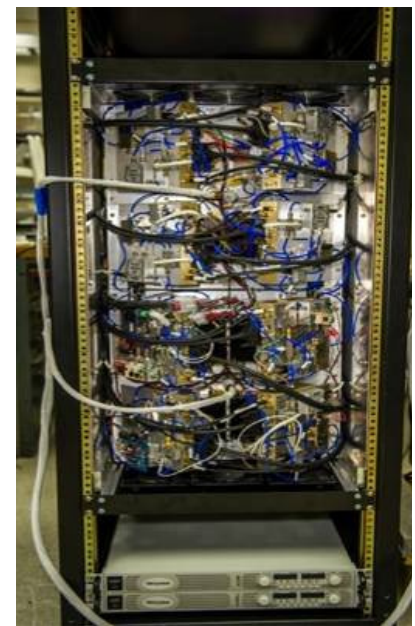

Fig. 4 EcoSAR Radar Electronics are installed on two aircraft racks (only one rack shown) located in the aircraft fuselage. 


\section{Radar Digital Unit (RDU)}

The instrument's RDU is responsible for multi-channel digital waveform generation, data acquisition, onboard processing, synchronization, REU timing, data transfer and archiving. The RDU's main core consists of four custom designed Vertix 6-based processor units. Each unit consists of 8 time-synchronous and phase-locked digital waveform synthesizers (with independent amplitude and phase control), and 8 independent data acquisition and receive channels. Each processor unit is dedicated to a polarization and side of the aircraft.. The four units are fully synchronized through a common trigger and local oscillator. The processor architecture is illustrated in Fig. 5, and a photograph of one the units is shown in Fig. 6.

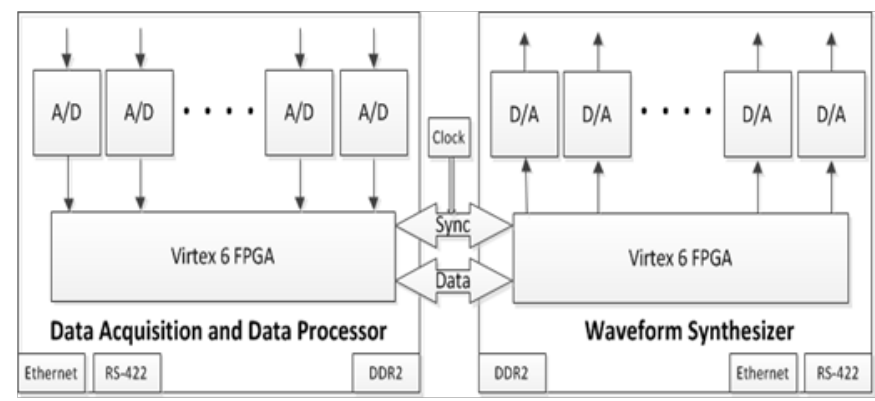

Fig. 5: EcoSAR's REU consists of custom FPGA-based digital electronics for waveform synthesis, data acquisition, real-time processor and archiving.

EcoSAR's transmit beam steering is performed at baseband using the RDU's multi-channel arbitrary waveform generation. Each of the channel's waveforms are synthesized with customized characteristics to generated the desired antenna beam pattern. On receive, the RDU's data acquisition section directly samples the RF signals and routes them to the onboard processer for down-conversion and filtering.

The waveform synthesizers and data acquisition and processors are controlled by custom Graphical Unit Interface (GUI) running on four Linux based servers which performs real-time configuration, monitoring, and data archiving. The RDU is designed to implement eight 10-Gbit Ethernet links for configuration, housekeeping, and data transfer to the host computers.

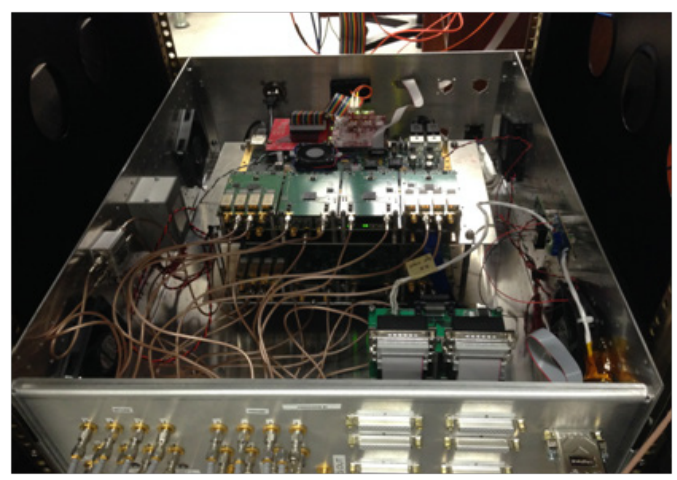

Figure 6: Each EcoSAR processor is made up of two Virtex 6-based programmable boards, and each dedicated to a polarization and side of the aircraft.

\section{SYSTEM CALIBRATION}

The EcoSAR system was tested and calibrated in a lowfrequency anechoic chamber prior to the flights. The calibration consisted in the characterization of all the radar channels in transmit and receive mode as a function of frequency, the generation of the steering weights, and the measurements of the transmit and receive co-polarized (co-pol) and cross-polarized (cross-pol) patterns. To measure EcoSAR cross-pol patterns, customized standard horns with high $(>30$ $\mathrm{dB}$ ) cross-pol isolation were designed for the chamber.

Figure 7 shows chamber measurements of the one-way "active" transmit patterns for the horizontal polarization at 435 MHz. Four across-track beams are shown corresponding to single sub-array (blue trace), full array - no amplitude taper steered to boresight (green trace), full array - cosine amplitude taper - steered to boresight (red trace), and cross polarization (magenta trace). Patterns for the vertical polarization were similar to the ones obtained for the horizontal polarization. All patterns agreed well with theoretical and simulated patterns.

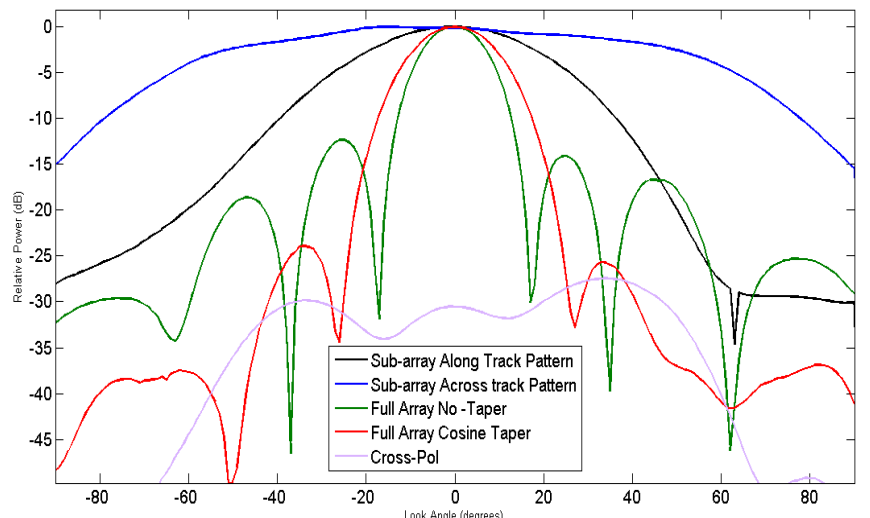

Figure 7 Measurements of EcoSAR's transmit patterns for the H-pol at 435 MHz.

Figure 8, shows digitally beamformed, one-way patterns, generated for the horizontal polarization at $435 \mathrm{MHz}$ in the anechoic chamber. These patterns agreed well with the transmit, theoretical and simulated patterns.

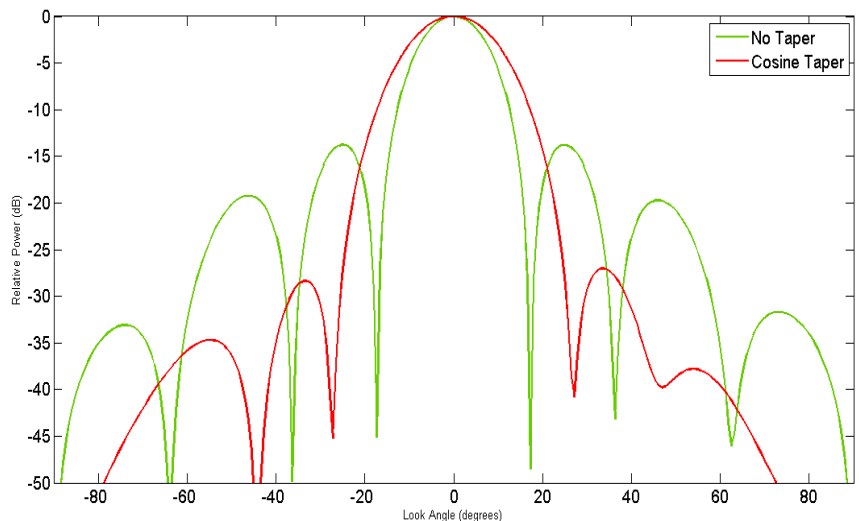

Figure 8 EcoSAR's digitally beamformed receive patterns for the H-pol at $435 \mathrm{MHz}$. 

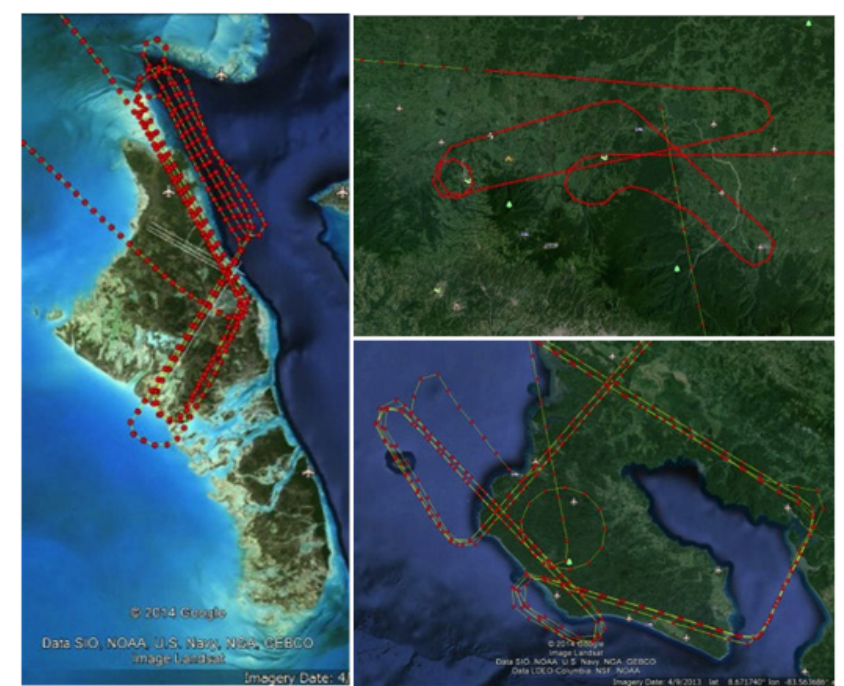

Fig. 9: EcoSAR's First flight campaign included areas of A) Andros Island, Bahamas; B) La Selva and C) Corcovado National Parks, Costa Rica. Red traces indicated flight tracks.

\section{FLIGHT CAMPAIGN}

EcoSAR's first flight campaign was conducted on NOAA's P3 aircraft between March 27 and March 31, 2014. The radar flew approximately 19 hours (including transit) over areas of Andros Island in the Bahamas, and the La Selva and the Corcovados National Parks in Costa Rica, as illustrated in Fig. 9.

Andros Island (Fig. 9A) was selected as a test site for its proximity to the aircraft base and for its variety of vegetation cover. The La Selva and the Corcovados National Parks (Fig. 9B and 9C) were selected for their high density forests. Field measurements at each of the sites were performed by the EcoSAR team during the same time frame as the flight campaign.

During the campaign, EcoSAR collected data at several bandwidths $(50 \mathrm{MHz}, 120 \mathrm{MHz}$, and $200 \mathrm{MHz}$ ) and at two altitudes $(3 \mathrm{~km}$ and $5 \mathrm{~km})$ in standard and Ping Pong modes. Examples of EcoSAR single pol (HH) and dual-pol (HH, HV) images are shown in Figs. 10 and 11, respectively. The images were acquired over Andros Island, Bahamas at an altitude of 3 $\mathrm{km}$ using the wide beam illumination technique [3].

Preliminary analysis of the data have indicated a successful instrument operation during the chamber calibration and first flight campaign. The analysis have also revealed the need of robust wideband beamforming and motion correction techniques to exploit the full system capability. The EcoSAR team is currently analyzing the entire calibration and campaign data sets, and developing the advanced imaging and calibration algorithms.

In addition, Radio Frequency Interference (RFI) identification and removal techniques are critical for the generation of high quality images. EcoSAR operates in the Pband which is routinely used by short-range hand held radios (walkie-talkies), remote controlled car and airplanes, cellular phones, and TV stations. These signals couple into the EcoSAR measurements, affecting image focusing and reducing image quality.
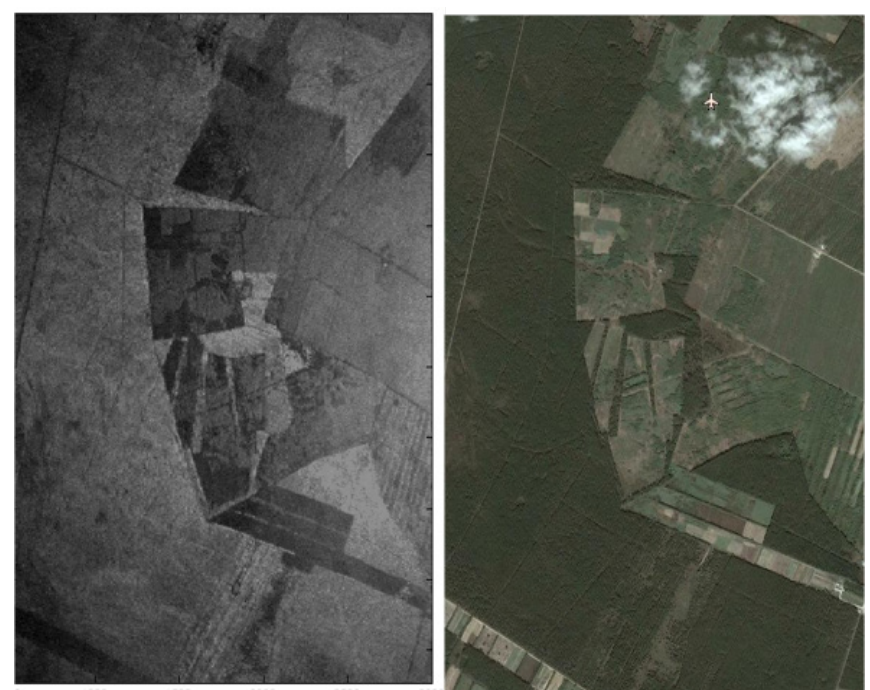

Fig. 10: EcoSAR images acquired on March 27, 2014 over forested areas on Andros Island, Bahamas. Left: $10 \mathrm{~m} \times 10 \mathrm{~m}$ resolution image at $\mathrm{HH}$ polarization. Right: Google areial of area imaged by EcoSAR.
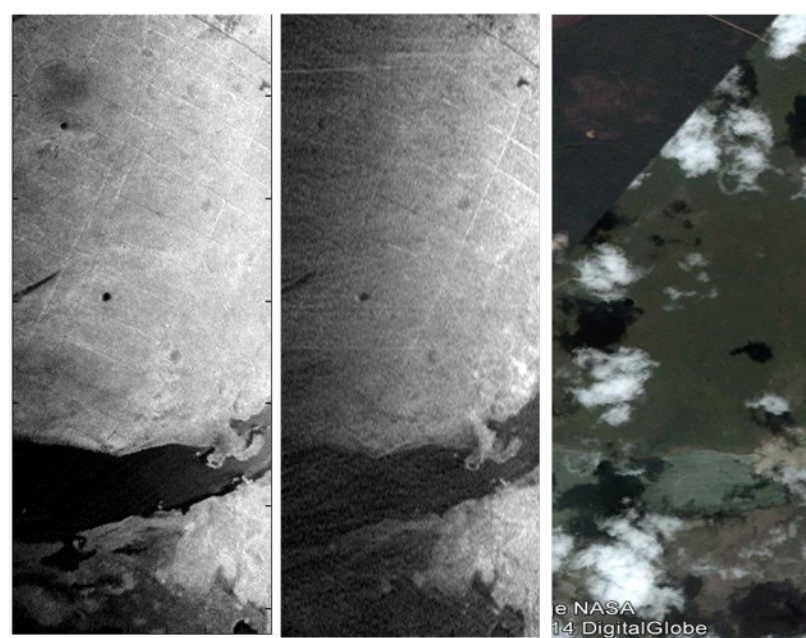

Fig. 11: Left and middle: $5 \mathrm{~m} \times 5 \mathrm{~m}$ reslotution, $\mathrm{HH}$ and $\mathrm{HV}$ polarization. Right: Google areial of area imaged by EcoSAR.

RFI measurements collected with EcoSAR in "sniffing mode" are shown in Fig. 12. Despite analog and digital filters and large coherent signal-processing gain of the SAR processing, RFI remains in the focused image. The RFI degradation can be seen in the HV-pol image shown in Fig. 11.

\section{CONCLUSION}

The EcoSAR's digital beamforming, polarimetric, and "single pass" interferometric radar was designed to support ecosystem structure and biomass research. The instrument was recently completed and flight tested over areas of the Bahamas and Costa Rica. The anechoic chamber calibration and the operation of the system during the campaign were successful and rich data sets were collected for analysis and algorithm development. 


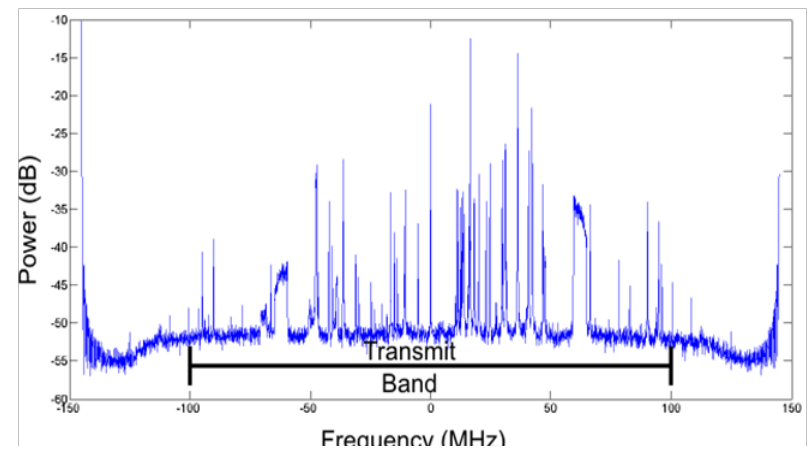

Figure 12 EcoSAR's "sniffing" acquisition mode shows the strong presence of RFI in the radar operational band. These unwanted signals can significantly degrade the quality of the SAR images and data products.

During the campaign, EcoSAR collected data at several bandwidths and altitudes in standard and Ping Pong modes. Preliminary analysis of the data have indicated a successful instrument operation during the chamber calibration and first flight campaign. The analysis have also indicated the need for the development of robust wideband beamforming and motion correction algorithms to exploit the full system capability. The EcoSAR team is currently analyzing and developing the required imaging and calibration algorithms.

\section{ACKNOWLEDGMENT}

The EcoSAR development and flight campaign was possible through a NASA Earth Science Technology (ESTO)
Instrument Incubator Program (IIP) award. Special thanks to the National Oceanic and Atmospheric Administration (NOAA) Aircraft Operations Center for the outstanding support in the integration of the EcoSAR instrument to the NOAA P3 aircraft and in the conduct of the EcoSAR flights.

\section{REFERENCES}

[1] Rincon,R, T. Fatoyinbo, G. Sun, K. J. Ranson, M. Perrine, M. Deshpande, Q. Bonds, 2011, The EcoSAR P-band SAR Synthetic Aperture Radar, Proc. IEEE Int. Geosci. Rem. Sens. Symp., July 25-29, 2011, Vancouver, Canada

[2] Fatoyinbo T., R. Rincon, G. Sun, K. J. Ranson, 2011, EcoSAR: A Pband Digital Beamforming Polarimetric Interferemetric SAR Instrument to Measure Ecosystem Structure and Biomass, Proc. IEEE Int. Geosci. Rem. Sens. Symp., July 25-29, 2011, Vancouver, Canada.

[3] Rincon, R. F.; Vega, M. A.; Buenfil, M.; Geist, A.; Hilliard, L.; Racette, P.; , "NASA's L-Band Digital Beamforming Synthetic Aperture Radar," Geoscience and Remote Sensing, IEEE Transactions on , vol.49, no.10, pp.3622-3628, Oct. 2011. doi: 10.1109/TGRS.2011.2157971.

[4] Earth Science and Applications from Space: National Imperatives for the Next Decade and Beyond. Committee on Earth Science and Applications from Space: A Community Assessment and Strategy for the Future, National Research Council (ESDS, 2007).

Responding to the Challenge of Climate and Environmental Change: NASA's Plan for a Climate-Centric Architecture for Earth Observations and Applications from Space, June 2010.

[5] R.Rincon, "Advanced Antenna for Digital Beamforming SAR", Earth Science Technolgoy Office (ESTO) Forum 2014, Oct 28-30, Leesburg, VA. (http://esto.nasa.gov/forum/estf2014/)

Hale, R.; Roberts, N., Liu, W., Yang, M., Schroer, D.; Kaushhik, B.; "Flight Safety Review: ECoSAR Integration on the NASA P-3". NASA internal document, June 2013. 